\title{
Experimental Intermittent Ischemia Augments Exercise-Induced Inflammatory Cytokine Production
}

Daniel D. Shill ${ }^{1}$, Kristine R. Polley ${ }^{1}$, T. Bradley Willingham ${ }^{1}$, Jarrod A. Call ${ }^{1}$, Jonathan R. Murrow $^{1,2}$, Kevin K. McCully ${ }^{1}$ FACSM, Nathan T. Jenkins ${ }^{1}$. ${ }^{1}$ University of Georgia, Athens, GA, ${ }^{2}$ Augusta University -- University of Georgia Medical Partnership, Athens, GA

Acute exercise-induced transient increases in inflammatory cytokines are linked to the beneficial vascular effects of exercise, but the underlying mechanisms that promote appearance are contingent on numerous factors (e.g. muscle mass recruited, exercise intensity/duration, etc.). Evidence suggests that a lack of oxygen and/or blood flow to working muscle modifies cytokine appearance. However, little is known about the inflammatory response to intermittent ischemia in working muscle. PURPOSE: Determine the extent to which local inflammation is involved in the response to ischemic exercise by reproducing the peripheral arterial disease (PAD)associated phenomenon of intermittent claudication without the presence of potential confounding comorbidities frequently exhibited by patients with PAD. METHODS: 14 healthy males performed unilateral isometric forearm contractions for 30 minutes with and without experimental ischemia. Blood was drawn at baseline, 5 and 10 minutes into exercise, at the end of exercise, and 30, 60, and 120 minutes after exercise. RESULTS: Oxygen saturation levels, as measured by near-infrared spectroscopy, were reduced by $10 \%$ and $41 \%$ during non-ischemic and ischemic exercise, respectively $(\mathrm{P}<0.001)$. Non-ischemic exercise did not affect cytokine values during exercise (all $\mathrm{P}>0.05$ ). Ischemic exercise enhanced concentrations of basic fibroblast growth factor, interleukin (IL)-6, IL-10, tumor necrosis factor-alpha, and vascular endothelial growth factor at the end of exercise by $148 \%, 197 \%, 129 \%, 154 \%$, and $164 \%(\mathrm{P}<$ $0.05)$, respectively, but IL-8 was not influenced by ischemic exercise $(\mathrm{P}>0.05)$.

CONCLUSION: In conclusion, the present study demonstrates that ischemic, small muscle endurance exercise elicits local inflammatory cytokine production, compared to non-ischemic exercise. The effect of ischemic exercise with PAD-associated comorbidities may impact the inflammatory response during and after exercise.

This study was supported by funds from the Office of the Vice President for Research and the College of Education at the University of Georgia (to N.T.J.). J.R.M. is Chief Operating Officer and K.K.M. is President of Infrared Rx. The other authors report no conflicts of interest, financial or otherwise. 